\title{
Combination of CT Imaging-based Radiomics and Clinicopathological Characteristics for Predicting the Clinical Benefit of Immune Checkpoint Inhibitors in Lung Cancer
}

\section{Bin Yang}

Nanjing University Medical School https://orcid.org/0000-0001-5518-7325

\section{Li Zhou}

Nanjing University Medical School Clinical College: East Region Military Command General Hospital Jing Zhong

Nanjing University Medical School Clinical College: East Region Military Command General Hospital Tangfeng Lv

Nanjing University Medical School Clinical College: East Region Military Command General Hospital Ang Li

Nanjing University Medical School Clinical College: East Region Military Command General Hospital Lu Ma

Nanjing University Medical School Clinical College: East Region Military Command General Hospital Jian Zhong

Nanjing University Medical School Clinical College: East Region Military Command General Hospital

\section{Saisai Yin}

Nanjing University Medical School Clinical College: East Region Military Command General Hospital

\section{Litang Huang}

Nanjing University Medical School Clinical College: East Region Military Command General Hospital

\section{Changsheng Zhou}

Nanjing University Medical School Clinical College: East Region Military Command General Hospital

\section{Xinyu Li}

Nanjing University Medical School Clinical College: East Region Military Command General Hospital

\section{YingQian Ge}

Siemens Healthcare China

\section{Xinwei Tao}

Siemens Healthcare China

\section{Longjiang Zhang}

Nanjing University Medical School Clinical College: East Region Military Command General Hospital

\section{Yong Song}

Nanjing University Medical School Clinical College: East Region Military Command General Hospital 


\section{Guangming Lu ( $\nabla$ cjr.luguangming@vip.163.com)}

Nanjing University Medical School Clinical College: East Region Military Command General Hospital

\section{Research}

Keywords: Non-small cell lung cancer, Radiomics, Machine learning, Immune checkpoint inhibitors, Survival outcome

Posted Date: November 11th, 2020

DOl: https://doi.org/10.21203/rs.3.rs-102368/v1

License: (c) (1) This work is licensed under a Creative Commons Attribution 4.0 International License. Read Full License 


\section{Abstract}

\section{Purpose}

In this study, we tested whether a combination of radiomic features extracted from baseline preimmunotherapy computed tomography (CT) images and clinicopathological characteristics could be used as novel noninvasive biomarkers for predicting the clinical benefits of non-small cell lung cancer (NSCLC) patients treated with immune checkpoint inhibitors (ICls).

\section{Methods}

Data on 92 consecutive patients with lung cancer who had been treated with ICls were retrospectively analyzed. In total, 88 radiomic features were selected from the pretreatment CT images for the construction of a random forest model. Radiomics model 1 was constructed based on the radscore.Through multivariate logistic regression analysis, the rad-score and significant predictors were integrated into a single predictive model (radiomics nomogram model 1) to predict the durable clinical benefit from ICls. Radiomics model 2 was developed based on the same rad-score as radiomics model 1 . Through multivariate Cox proportional hazards regression analysis using the rad-score and independent risk factors, radiomics nomogram model 2 was constructed to predict the progression-free survival (PFS).

Results

The models successfully predicted the patients who would benefit from ICls. With radiomics model 1 , the area under the receiver operating characteristic curve values for the training and validation cohorts were 0.848 and 0.795 , respectively, whereas with radiomics nomogram model 1 , the values were 0.902 and 0.877 , respectively. For the PFS prediction, the Harrell's concordance indexes for the training and validation cohorts were 0.717 and 0.760 , respectively, with radiomics model 2 , whereas they were 0.749 and 0.791 , respectively, with radiomics nomogram model 2 .

\section{Conclusion}

CT-based radiomic features and clinicopathological factors can be used prior to the initiation of immunotherapy for identifying NSCLC patients who are the most likely to benefit from the therapy. This could be a step forward toward the realization of precision medicine and individualized decision support for patients with advanced NSCLC.

\section{Introduction}

According to the latest epidemiological data, lung cancer has become the most common of the malignant cancer types, with the highest morbidity and mortality rates worldwide [1]. Among the common subtypes of lung cancer, non-small cell lung cancer (NSCLC) accounts for more than $85 \%$ of patients with lung cancer [2]. Despite significant advances in cancer diagnosis and treatment techniques, the long-term survival rate of patients with lung cancer is only $10 \%-15 \%$, and once metastasis occurs, the 5 -year 
survival rate is less than 5\% [3]. Therefore, enhancing the efficacy of treatments is key to improving the overall prognosis of patients with advanced NSCLC.

Although cytotoxic therapy remains an important component of systemic therapy, many chemotherapy regimens are associated with significant toxic effects [4]. Compared with traditional cytotoxic agents, tyrosine kinase inhibitors (TKIs) have led to more favorable outcomes and have thus become the first-line treatment for patients with actionable driver mutations [5]. However, there are still many patients who do not have genetic mutations and would therefore not benefit from TKIs [6]. For such patients, immune checkpoint inhibitors (ICls) have become a good option, particularly antibodies targeting the programmed cell death-1 (PD1) - programmed cell death ligand-1 (PD-L1) axis, which can restore the body's antitumor immune response by blocking the immune suppression signals between the tumor and T cells [7]. The ICls that are approved or in development for the treatment of lung cancer are the anti-PD1 antibodies nivolumab and pembrolizumab and the anti-PD-L1 antibodies atezolizumab, avelumab, and durvalumab [8]. Intriguingly, the uses of ICls have shifted from the advanced stage to earlier stages of lung cancer in an attempt to reach the ultimate goal of curing the disease completely [9].

However, despite the substantial progress made in immunotherapy research and development, the majority of patients still do not derive benefits from ICls [10]. Currently, PD-L1 and microsatellite instability/defective mismatch repairs are approved for clinical use as predictive biomarkers of a tumor's response to ICls [11]. Additionally, multicohort studies such as CheckMate 026 have shown that although a high tumor mutation burden is positively correlated with the efficacy of ICls [12], this parameter is not a perfect biomarker for the selection of patients for $\mathrm{ICl}$ therapy [13]. Hence, there is an important and urgent need to identify and develop predictive biomarkers for immunotherapy. Moreover, an increasing number of studies have pointed out that combining different biomarkers to reduce the assumptive risk associated with each one can improve the performance of the ultimate predictive biomarker for ICls [14].

Medical imaging is a noninvasive inspection method with prognostic value. The quantitative imaging features extracted from conventional medical images can be used to predict the clinical prognosis or monitor the treatment response of different types of cancer [15-17]. Radiomics is a quantitative research method that involves the conversion of image data into high-throughput image feature data that can then be mined and used to describe the intensity, shape, and texture of tumors and quantify the heterogeneity of time and space of tumor tissue. Essentially revealing disease features that cannot be recognized by the naked eye. The technique effectively transforms images into a high-dimensional recognizable feature space and uses statistical and/or machine learning methods to select the most valuable radiomic features for analyzing clinical information. The end goal is to construct a model with diagnostic, prognostic, or predictive value that will provide valuable information for enabling the accurate individualized diagnosis and treatment of diseases [18-20].

The nomogram is a graphical diagram that is easy to interpret and contains different kinds of predictors. In recent years, it has become an important tool for cancer research experts [21, 22]. In several studies, computed tomography (CT) or positron emission tomography (PET/CT)-based radiomics has been used 
to improve the prediction of the performance of various cancers, and it has been found that the combination of radiomic features with the traditional tumor staging system and other clinicopathological factors may improve the accuracy of tumor prognosis prediction [23-25]. Overall, however, the prediction performances of current prognostic prediction models are generally not very good [26-28].

Therefore, in this study, radiomics nomogram models for predicting the tumor response and prognosis of patients with NSCLC following ICl treatment were constructed on the basis of CT radiomic features and clinicopathological characteristics. The ultimate aim is to develop prediction models that can guide individualized immunotherapy in patients with lung cancer.

\section{Methods}

\section{Patients}

Data were collected on 149 patients with lung cancer who were treated at the Affiliated Jinling Hospital, Medical School of Nanjing University between June 2015 and December 2019. The institutional review board of the Affiliated Jinling Hospital, Medical School of Nanjing University approved this retrospective study and waived the requirements for informed consent from the patients. However, consent was obtained from the patients on the publishing of their images and clinical information. The inclusion criteria were as follows: patients of 18 years of age or older; a diagnosis of lung cancer, as confirmed by histopathology according to the eighth edition of the American Joint Commission on Cancer TNM classification and staging system; and a recipient of immunotherapy for the cancer. The exclusion criteria were as follows: patients with missed follow-up $(n=20)$; patients with a treatment duration of less than 6 months before progressive disease (PD) $(n=25)$; and patients with partial loss of images $(n=12)$. In total, 94 patients met the inclusion criteria and were randomly divided at a 7:3 ratio into the training cohort $(n=64)$ and the validation cohort $(n=28)$ (Figure 1). For each patient, baseline clinicopathological characteristics were obtained from their medical records; namely, age, sex, smoking status, family history, histological subtype, TNM classification and staging, blood cell counts (platelets, white blood cells, neutrophils, lymphocytes, and monocytes), and levels of thyroid transcription factor 1 (TTF-1), Ki-67, Creactive protein (CRP), carcinoembryonic antigen, and neuron-specific enolase. The time from the beginning of immunotherapy to the date of disease progression was defined as progression-free survival (PFS). The endpoint of this study was the clinical benefit of immunotherapy, which was defined as either a durable clinical benefit (DCB: complete response, partial response (PR), or stable disease (SD) lasting $>6$ months) or no durable clinical benefit (NDB: PD or SD that lasted $\leq 6$ months).

\section{Image acquisition}

All patients underwent non-enhanced CT imaging of the lungs with one of three multidetector row CT systems (SOMATOM Definition Flash, SOMATOM Emotion, and SOMATOM Perspective, all from Siemens Healthineers AG, Erlangen, Germany). After removing any metallic foreign bodies from the chest area, the patient was placed in the supine position with both hands raised. The CT scan was conducted using the spiral scanning mode and ranged from the thoracic entrance to the underlying layer of the lung, with a 
single breath-hold scan at the end of inspiration. The following CT acquisition parameters were used: 120 or $130 \mathrm{kVp} ; 160 \mathrm{mAs}$; detector collimation: $6 \times 1.25 \mathrm{~mm}, 64 \times 0.625 \mathrm{~mm}$, or $64 \times 0.6 \mathrm{~mm}$; rotation time: 0.5 or 0.8 s; matrix size: $512 \times 512$; field of view: $350 \times 350 \mathrm{~mm}$ ). Each patient received a whole-lung scan, and the CT image retrieved from the picture archiving and communication system was reconstructed with a standard kernel. The slice thickness of the CT images ranged between 1 and 1.25 $\mathrm{mm}$.

\section{Image segmentation and radiomic feature extraction}

This study followed and adhered to the Image Biomarker Standardization Initiative (IBSI) guidelines [29] and the radiomics prototype software program used (Radiomics, Frontier, Siemens) was IBSI compliant. A volume of interest was drawn semiautomatically around the tumor by a chest radiologist (Y.B., 9 years of experience) in the lung diagnosis using Radiomics and confirmed by another chest radiologist (Z.J.,15 years of experience). Both radiologists were blinded to the patients' clinical information. First, we imported the CT images into Radiomics. In the segmentation module of Radiomics, a few segmentation tools are available for the semiautomatic delineation of the tumor in three dimensions. The segmentation is semiautomatically produced by drawing a line across the boundary of the tumor. Then, through an automatic algorithm, the tool finds neighboring voxels with the same gray level in three-dimensional (3D) space, generating random walker-based lesion segmentation for solid and subsolid lung lesions [30]. If the segmentation is incorrect, the operators could correct it manually in the 3D domain using the Radiomics prototype. As a result, a total of 111 features (viz., 18 first-order, 75 texture, and 18 size and shape features) were extracted from the CT images using Radiomics. To test the intraclass reproducibility, the data for 25 randomly selected patients were segmented twice by one radiologist (Y.B.) within a 1-month period. To test the interclass reproducibility, the same 25 sets of data were segmented by two radiologists (Y.B. and Z.J.). Spearman correlation analysis was used to assess the differences between the features generated at different times and by different radiologists as well as between the twice-generated features by the same radiologist. Interclass and intraclass correlation coefficients (ICCs) were used to evaluate the intra- and inter-observer agreement of feature extraction, where an ICC value of greater than 0.80 indicated good agreement. As a result, 88 features were retained for further analysis (Figure 2).

\section{Predictive model construction and model testing}

A model for predicting the clinical benefits of immunotherapy was constructed on the basis of the CTderived radiomic features, using the random forest (RF) method. Patients with DCB were labeled as positive and those with NDB as negative. The RF algorithm has a comparably low tendency to overfit and is well suited for datasets with a large number of heterogeneous predictors and cluster-correlated observations; thus, it was adopted for the machine learning-based prediction model. The RF method was used to construct the prediction model because of its high variance-bias trade-off capability. It is a classification algorithm consisting of many decision trees, where each tree represents a weak classifier. A combination of trees can achieve improved model performance. The split quality was measured 
according to the Gini impurity. Hyperparameter optimization was performed. The RF model was evaluated with an additional independent cohort study, which was randomly selected with potentially unseen test patients. The performance of the model was assessed on the basis of its receiver operating characteristic (ROC) curve, area under the ROC curve (AUC), accuracy, sensitivity, and specificity. Finally, radiomics model 1 was established on the basis of 15 important radiomic features (Figure 3A). The forest consisted of five trees, and the maximum depth of the tree was set as 2 . Three features were considered when looking for the best split. Finally, a radiomics score (rad-score) was output for each patient.

\section{Clinicopathological factors plus radiomics model development and radiomics nomogram model construction}

The clinicopathological factors were analyzed using univariate logistic regression analysis, in which the predictors with a $p$ value of less than 0.10 were included to find those of significance. Through multivariate logistic regression analysis, the multimodal features,rad-score and significant predictors were then integrated into a single predictive model, whereupon radiomics nomogram model 1 was constructed for the training cohort. Calibration curves were plotted to assess the calibration of radiomics nomogram model 1. Decision curve analysis was conducted to determine the clinical usefulness of the radiomics model and radiomics nomogram model by quantifying the net benefits at different threshold probabilities for the training and validation cohorts.

\section{Clinicopathological factor analysis}

The clinicopathological factors were analyzed using univariate Cox proportional hazards (CPH) regression analysis. Those factors with a $p$ value of less than 0.10 and the rad-score were then combined for multivariate $\mathrm{CPH}$ regression analysis to identify the independent risk factors, which were subsequently analyzed using the Kaplan-Meier curve and log-rank test. The final model was selected by backward stepwise elimination, with the Akaike information criterion as the stopping rule [31].

\section{Construction of radiomics nomogram model 2}

The same rad-score was used to construct radiomics model 2. The multimodal features and parameters, including the rad-score and independent risk factors, were integrated into a single predictive model through multivariate $\mathrm{CPH}$ regression analysis, where upon radiomics nomogram model 2 was developed for the training cohort. The prognostic abilities of the generated models were evaluated using the training cohort and validated using the validation cohort. The discrimination performance of the prognostic models was assessed using Harrell's concordance index (C-index), which ranges between 0.5 (indicating a random distribution of the data) and 1.0 (indicating perfect prediction of the observed survival information by the model). Calibration curves of the nomogram were subsequently drawn for the 2-year PFS of the patients. The calibration curves were used to determine the independent risk factors as well as to indicate both the PFS probabilities predicted by the prognostic models and the observed probabilities. 


\section{Statistical analysis}

The differences in clinicopathological factors between the training and validation datasets were assessed using the Mann-Whitney $U$ test for continuous variables and the $\chi^{2}$ test for categorized variables. The discrimination power of the models was measured from the AUC and the C-index. The Delong test was used to compare two AUCs, and the log-likelihood ratio was used to assess the increase in predictive power of the C-index. The radiomics nomogram model 2 score was calculated and used to assess risk stratification. Survival curves were generated with the Kaplan-Meier method and compared using the two-sided log-rank test. The optimal cutoff point for continuous prognostic markers in the survival analysis was determined using X-tile software (version 3.6.1; Yale University School of Medicine, New Haven, CT, USA). A calibration curve was generated to demonstrate the goodness of fit, which is a graphical representation of the relationship between observed and predicted survival. The GreenwoodNam-D'Agostino (GND) method was applied to measure the statistical significance of the goodness-of-fit test results. The prediction error of the models, which was assessed using the "Boot632plus" split method with 100 iterations to calculate estimates of the prediction error curves, was summarized as the integrated Brier score, which represents a valid measure of the overall model performance and can range from 0 (for a perfect model) to 0.25 (for a noninformative model with a $50 \%$ incidence of the outcome). The RF model was conducted using Python software (Python Scikit-learn package comprising Python version 3.7 and Scikit-learn version 0.21 ; http://scikit-learn.org/). The construction of radiomics nomogram model 2, assessment of the model, and decision curve analysis(DCA) were performed using $\mathrm{R}$ software (version 3.4.4; http://www.r-project.org). All the codes are available at https://github.com/tomato08217/immune. All statistical tests were two-sided, with a significance level of 0.05 .

\section{Results}

\section{Clinicopathological characteristics of the patients}

The baseline clinical characteristics of the patients in the training and validation cohorts are listed in Table 1. There were no significant differences in sex, age, smoking status, family history, histology type, cancer stage, TTF-1, and Ki-67 ( $p=0.070-1.000)$ between the two cohorts.

\section{Prognostic model performance and development of radiomics nomogram model 1}

In total, 88 CT-derived radiomic features were used to build radiomics model 1. Because the logistic regression analysis had identified the rad-score, age, stage $n 1$, stage $n 2$, stage $n 3$, and stage $M$ as being independent predictors (Table 2), they were used to build the model, which was designated as radiomics nomogram model 1. After 10 -fold cross-validation, radiomics model 1 was found to have an AUC value of 0.848 , a sensitivity value of 0.625 , a specificity value of 0.906 , and an accuracy value of 0.766 for the training cohort, whereas it had an AUC value of 0.795 , a sensitivity value of 1.000 , a specificity value of 
0.704 , and an accuracy value of 0.714 for the validation cohort (Figure 3B). For radiomics nomogram model 1 , the AUC value was 0.902 , the sensitivity value was 0.857 , the specificity value was 0.884 , and the accuracy value was 0.875 for the training cohort, whereas the AUC was 0.877 , the sensitivity value was 0.800 , the specificity value was 0.944 , and the accuracy value was 0.893 for the validation cohort (Figure 3Cand Table 3). The independent predictors were presented as radiomics nomogram model 1 (Figure 4A). The calibration curve for the probability of DCB and NDB in the training and validation cohorts demonstrated good agreement between the predicted and actual values (Figure 4B). The Brier scores also showed good performance of the model, with values of 0.178 for the train dataset and 0.187 for the test dataset. Decision curve analysis was performed to determine the clinical usefulness of radiomics nomogram model 1 by quantifying the net benefits at different threshold probabilities. The results showed that radiomics nomogram model 1 had a higher overall net benefit than radiomics model 1 across the majority of the range of reasonable threshold probabilities (Figure 4C).

\section{Clinicopathological factor analysis}

The clinicopathological factors were analyzed using univariate $\mathrm{CPH}$ regression analysis to test the hazard ratio of each factor and to determine its significance in the probability of PFS. The factors with a $p$ value of less than 0.10 were then combined for multivariate $\mathrm{CPH}$ regression analysis to identify independent risk factors, whereupon the rad-score, CRP level, and $\mathrm{M}$ stage were found to be the independent risk factors for the patients (Table 4). These independent risk factors were then analyzed using the Kaplan-Meier curve and log-rank test, with the latter indicating significant discrimination between the two groups. Figure 5A-C shows the PFS probability of the patients in the high-risk or low-risk cohorts.

\section{Prognostic prediction model performance and development of radiomics nomogram model 2}

For radiomics model 2, the C-indexes were 0.717 and 0.760 for the training and validation cohorts, respectively, whereas for radiomics nomogram model 2 , the values were 0.749 and 0.791 , respectively (Table 5). The independent risk factors were presented as the nomogram (Figure 6A). The calibration curve showed that the predicted probability was significantly close to the actual PFS of patients, with $p=$ 0.21 in the GND goodness-of-fit test (Figure 6B). The Brier scores also showed good performance of the model, with values of 0.187 for the train dataset and 0.209 for the test dataset. The decision curve analysis of the clinical usefulness of radiomics nomogram model 2 showed that the model had a higher overall net benefit than radiomics model 2 across the majority of the range of reasonable threshold probabilities (Figure 6C).

\section{Discussion}

In this study, an RF model based on the radiomic features of CT images and a radiomics model (radiomics model 1) based on the rad-score were established, following which multivariate logistic regression analysis of the rad-score combined with clinicopathological factors was carried out to construct a radiomics nomogram model (radiomics nomogram model 1) for distinguishing patients who 
received DCB from those who received NDB after ICI treatment. Then, a second radiomics model (radiomics model 2) based on the rad-score was established, following which multivariate $\mathrm{CPH}$ regression analysis of the rad-score combined with independent risk factors was conducted to establish a second radiomics nomogram model (radiomics nomogram model 2) for predicting PFS after immunotherapy. The ultimate goals are to use these models to identify patients who can benefit from immunotherapy and to provide both guidance for individualized immunotherapy and a reference for the advancement of precision medicine.

Multivariate logistic regression analysis of the rad-score and significant clinicopathological factors combined showed that the rad-score, age, stage $n 1$, stage $n 2$, stage $n 3$, and stage $M$ were independent predictors, among which all (except age and stage $\mathrm{n} 1$ ) can benefit from immunotherapy. The prediction models built had good prediction performances. Radiomics model 1 had an AUC value of 0.848 , a sensitivity value of 0.625 , a specificity value of 0.906 , and an accuracy value of 0.766 for the training cohort, whereas it had an AUC value of 0.795 , a sensitivity value of 1.000 , a specificity value of 0.704 , and an accuracy value of 0.714 for the validation cohort. The independent predictors (rad-score, age, stage n1, stage $n 2$, stage $n 3$, and stage $M$ ) were used to build radiomics nomogram model 1 , the performance of which was good, with an AUC value of 0.902 , a sensitivity value of 0.857 , a specificity value of 0.884 , and an accuracy value of 0.875 in the training cohort, and an AUC value of 0.877 , a sensitivity value of 0.800 , a specificity value of 0.944 , and an accuracy value of 0.893 in the validation cohort. Mu et al. [32] conducted radiomics of pretreatment ${ }^{18} \mathrm{~F}$-FDG PET/CT images to predict the clinical benefit of checkpoint blockade immunotherapy for patients with advanced NSCLC. Their results showed that the multiparametric radiomics signature could predict the patients who received $D C B$, with AUC values of $0.86(95 \% \mathrm{Cl} 0.79-0.94), 0.83(95 \% \mathrm{Cl} 0.71-0.94)$, and 0.81 (95\% $\mathrm{Cl} 0.68-0.92)$ for the training, retrospective test, and prospective test cohorts, respectively. Trebeschi et al. [33] performed an artificial intelligencebased characterization of each lesion on pretreatment contrast-enhanced CT imaging data to develop and validate a noninvasive machine learning biomarker for distinguishing between anti-PD1 immunotherapy responding and non-responding patients with advanced melanoma and NSCLC. Their results showed that the biomarker achieved significant performance for NSCLC lesions (AUC $=0.83, p<$ 0.001 ) and borderline significance for melanoma lymph nodes (AUC $=0.64, p=0.05$ ). After combining these lesion-wide predictions at the patient level, the immunotherapy response could be predicted with an AUC value of up to 0.76 for both cancer types $(p<0.001)$. Thus, the performance of our prediction model is more encouraging than that of previous studies, with the possible reasons being as follows. First, it may be attributed to our implementation of a cross-validation approach and uses of a performancedriven feature selection strategy and the RF algorithm for model training (which is robust to overfitting) to build a reliable model with higher performance. These strategies have been proposed in previous studies to achieve better model performance [34,35]. Additionally, independent predictors were selected to construct the models, and combining these clinical predictors may have improved the model performance. 
Moreover, the same rad-score was used to construct radiomics model 2, the C-indexes of which were 0.717 and 0.760 for the training and validation cohorts, respectively. The rad-score was combined with significant factors $(p<0.10)$ for multivariate $\mathrm{CPH}$ regression analysis to identify independent risk factors, whereupon the rad-score, CRP level, and M stage were identified as such for patients. It was demonstrated that patients with a CRP level greater than or equal to 1.22 and stage M1 cancer were more likely to progress positively after receiving immunotherapy. Radiomics nomogram model 2 was established using the same three independent risk factors and the C-indexes were 0.749 and 0.791 for the training and validation cohorts, respectively. Our model has better predictive performance than that of previous studies [32].The independent predictors and independent risk factors were presented as nomograms, where the calibration curves for the probability of response prediction or survival analysis demonstrated good agreement between prediction and observation. Moreover, the decision curve analysis showed that the radiomics nomogram models had a higher overall net benefit than the radiomics models across the majority of reasonable threshold probabilities. This demonstrates that our models not only have better predictive performance than that of other studies but are also more robust and stable.

To our best knowledge, this is the first study to have used the noninvasive radiomics approach based on CT images and clinicopathological characters to predict the efficacy of ICls in Chinese patients with lung cancer. Our study has some limitations; namely, the relatively small sample size due to the relatively late date of approval of ICls (in 2018) by the China Food and Drug Administration; the use of only a singlecenter cohort; the retrospective nature of the data; and the lack of external validation, which may have introduced selection bias. However, we plan to rapidly expand the sample size and recruit multicenter cohorts for validation of the models in the near future. Additionally, we did not conduct research on overall survival but will add this in future studies.

In conclusion, by combining CT image-based radiomics and clinicopathological factors, radiomics nomogram models were constructed for identifying patients with NSCLC who would most likely benefit from immunotherapy and for assessing their PFS post therapy. The good performances of the models suggest that they could be used to provide more precise guidance for the accurate diagnosis and treatment of NSCLC in clinical practice.

\section{Abbreviations}

Cl: confidence interval; C-index: Harrell's concordance index; NSCLC: non-small cell lung cancer; TNM: tumor-node-metastasis; TKIs: tyrosine kinase inhibitors; ICls: immune checkpoint inhibitors; CRP: Creactive protein; PFS: progression-free survival; DCB: durable clinical benefit; PR: partial response; SD: stable disease; NDB: no durable clinical benefit; ROC: receiver operating characteristic; AUC: area under the receiver operating characteristic curve; $\mathrm{CPH}$ : Cox proportional hazards.

\section{Declarations}

\section{Acknowledgments}


The authors thank all the personnel from the Department of Medical Imaging, Affiliated Jinling Hospital, Medical School of Nanjing University for their collective efforts on the image post-processing, clinical management, and follow-up work.

\section{Author contributions}

BY conceived the study. BY, LZ, J Zh, TF-L,AL, J Zh, LM, SS-Y, XY-L, LT-H, and CSh-Z collected the data. TF$L, L J-Z$ and GM-L performed the image analysis. BY and LZ wrote the manuscript. YQ-G and XW-T performed the statistical analyses. YQ-G, LJ-Z, GM-L, and YS edited and reviewed the manuscript. All the authors discussed the results and commented on the manuscript.

\section{Financial support}

This work was supported by the National Key Research and Development Program of China (2017YFC0113400 for LJZ).

\section{Availability of data}

Datasets are available on request. The raw data supporting the conclusions of this manuscript will be made available by the authors, without undue reservation, to any qualified researcher. Requests to access the datasets should be directed to Dr. Guangming Lu (E-mail: cjr.luguangming@vip.163.com).

\section{Competing Interests}

The authors have declared that no competing interest exists.

\section{References}

1. Siegel RL, Miller KD, Jemal A. Cancer statistics, 2019. CA Cancer J Clin. 2019; 69: 7-34.

2. Herbst RS, Morgensztern D, Boshoff $C$. The biology and management of non-small cell lung cancer. Nature. 2018; 553: 446-54.

3. Fidler MM, Bray F, Soerjomataram I. The global cancer burden and human development: a review. Scand J Public Health. 2018; 46: 27-36.

4. Griesinger F, Korol EE, Kayaniyil S, Varol N, Ebner T, Goring SM. Efficacy and safety of first-line carboplatin-versus cisplatin-based chemotherapy for non-small cell lung cancer: a meta-analysis Lung Cancer. 2019; 135: 196-204.

5. Recondo G, Facchinetti F, Olaussen KA, Besse B, Friboulet L. Making the first move in EGFR-driven or ALK-driven NSCLC: first-generation or next-generation TKI? Nat Rev Clin Oncol. 2018; 15: 694-708.

6. Hirsch FR, Scagliotti GV, Mulshine JL, Kwon R, Curran WJ Jr, Wu YL, et al. Lung cancer: current therapies and new targeted treatments. Lancet. 2017; 389: 299-311.

7. Velcheti V, Schalper K. Basic overview of current immunotherapy approaches in cancer. Am Soc Clin Oncol Educ Book. 2016; 35: 298-308. 
8. Sanmamed MF, Chen LP. A paradigm shift in cancer immunotherapy: from enhancement to normalization. Cell. 2018; 175: 313-26.

9. Ghysen K, Vansteenkiste J. Immunotherapy in patients with early stage resectable nonsmall cell lung cancer. Curr Opin Oncol. 2019; 31: 13-7.

10. Kelderman S, Schumacher TN, Haanen JB. Acquired and intrinsic resistance in cancer immunotherapy. Mol Oncol. 2014; 8: 1132-9.

11. Duffy MJ, Crown J. Biomarkers for predicting response to immunotherapy with immune checkpoint inhibitors in cancer patients. Clin Chem. 2019; 65: 1228-38.

12. Peters $S$, Creelan B, Hellmann MD, Socinski MA, Reck M, Bhagavatheeswaran P, et al. Impact of tumor mutation burden on the efficacy of first-line nivolumab in stage IV or recurrent non-small cell lung cancer: an exploratory analysis of CheckMate 026 [Abstract]. Cancer Res. 2017; 77 (Suppl. 13): СТ082.

13. Liu SY, Wu YL. Biomarker for personalized immunotherapy. Transl Lung Cancer Res. 2019; 8 (Suppl. 3): S308-17.

14. Camidge DR, Doebele RC, Kerr KM. Comparing and contrasting predictive biomarkers for immunotherapy and targeted therapy of NSCLC. Nat Rev Clin Oncol. 2019; 16: 341-55.

15. Zhang H, Graham CM, Elci O, Griswold ME, Zhang X, Khan MA, et al. Locally advanced squamous cell carcinoma of the head and neck: CT texture and histogram analysis allow independent prediction of overall survival in patients treated with induction chemotherapy. Radiology. 2013; 269: 801-9.

16. Panth KM, Leijenaar RT, Carvalho S, Lieuwes NG, Yaromina A, Dubois L, et al. Is there a causal relationship between genetic changes and radiomics-based image features? An in vivo preclinical experiment with doxycycline inducible GADD34 tumor cells. Radiother Oncol. 2015; 116: 462-6.

17. Coroller TP, Grossmann P, Hou Y, Rios Velazquez E, Leijenaar RT, Hermann G, et al. CT-based radiomic signature predicts distant metastasis in lung adenocarcinoma. Radiother Oncol. 2015; 114: 345-50.

18. Lambin P, Rios-Velazquez E, Leijenaar R, Carvalho S, van Stiphout RG, Granton P, et al. Radiomics: extracting more information from medical images using advanced feature analysis. Eur $\mathrm{J}$ Cancer. 2012; 48: 441-6.

19. Lambin P, van Stiphout RG, Starmans MH, Rios-Velazquez E, Nalbantov G, Aerts HJ, et al. Predicting outcomes in radiation oncology - multifactorial decision support systems. Nat Rev Clin Oncol. 2013; 10: $27-40$.

20. Fan M, Zhang P, Wang Y, Peng W, Wang S, Gao X, et al. Radiomic analysis of imaging heterogeneity in tumours and the surrounding parenchyma based on unsupervised decomposition of DCE-MRI for predicting molecular subtypes of breast cancer. Eur Radiol. 2019; 29: 4456-67.

21. Tang XR, Li YQ, Liang SB, Jiang W, Liu F, Ge WX, et al. Development and validation of a gene expression-based signature to predict distant metastasis in locoregionally advanced nasopharyngeal carcinoma: a retrospective, multicentre, cohort study. Lancet Oncol. 2018;19: 382-93.

22. Tanadini-Lang S, Rieber J, Filippi AR, Fode MM, Streblow J, Adebahr S, et al. Nomogram based overall survival prediction in stereotactic body radiotherapy for oligo-metastatic lung 
disease. Radiother Oncol. 2017; 123: 182-8.

23. Guo W, Li H, Zhu Y, Lan L, Yang S, Drukker K, et al. Prediction of clinical phenotypes in invasive breast carcinomas from the integration of radiomics and genomics data. J Med Imaging (Bellingham). 2015; 2: 041007.

24. Zhang B, Tian J, Dong D, Gu D, Dong Y, Zhang L, et al. Radiomics features of multiparametric MRI as novel prognostic factors in advanced nasopharyngeal carcinoma. Clin Cancer Res. 2017; 23: 425969.

25. Parmar C, Leijenaar RT, Grossmann P, Rios Velazquez E, Bussink J, Rietveld D, et al. Radiomic feature clusters and prognostic signatures specific for Lung and Head \& Neck cancer. Sci Rep. 2015; 5: 11044.

26. de Jong EEC, van Elmpt W, Rizzo S, Colarieti A, Spitaleri G, Leijenaar RTH,et al. Applicability of a prognostic CT-based radiomic signature model trained on stage I-III non-small cell lung cancer in stage IV non-small cell lung cancer. Lung Cancer. 2018; 124: 6-11.

27. Aerts HJ, Velazquez ER, Leijenaar RT, Parmar C, Grossmann P, Carvalho S, et al. Decoding tumour phenotype by noninvasive imaging using a quantitative radiomics approach. Nat Commun. 2014; 5: 4006.

28. Parmar C, Grossmann P, Rietveld D, Rietbergen MM, Lambin P, Aerts HJ. Radiomic machine-learning classifiers for prognostic biomarkers of head and neck cancer. Front Oncol. 2015; 5: 272.

29. Zwanenburg A, Vallières M, Abdalah MA, Aerts HJWL, Andrearczyk V, Apte A, et al. The image biomarker standardization initiative: standardized quantitative radiomics for high-throughput imagebased phenotyping. Radiology. 2020; 295: 328-38.

30. Wels MG, Lades F, Muehlberg A, Suehling M. General purpose radiomics for multi-modal clinical research. In: Mori K, Hahn HK, Ed. Proceedings of SPIE Medical Imaging 2019: Computer-Aided Diagnosis, 16-21 February 2019, San Diego, California, USA. DOI: 10.1117/12.2511856.

31. Akaike H. A new look at the statistical model identification. IEEE Trans Automat Contr. 1974; 19: 71623.

32. Mu W, Tunali I, Gray JE, Qi J, Schabath MB, Gillies RJ. Radiomics of ${ }^{18}$ F-FDG PET/CT images predicts clinical benefit of advanced NSCLC patients to checkpoint blockade immunotherapy. Eur $\mathrm{J}$ Nucl Med Mol Imaging. 2020; 47: 1168-82.

33. Trebeschi S, Drago SG, Birkbak NJ, Kurilova I, Cǎlin AM, Del li Pizzi A, et al. Predicting response to cancer immunotherapy using noninvasive radiomic biomarkers. Ann Oncol. 2019; 30: 998-1004.

34. Yang X, Dong X, Wang J, Li W, Gu Z, Gao D, et al. Computed tomography-based radiomics signature: a potential indicator of epidermal growth factor receptor mutation in pulmonary adenocarcinoma appearing as a subsolid nodule. Oncologist. 2019; 24: e1156-64.

35. Jia TY, Xiong JF, Li XY, Yu W, Xu ZY, Cai XW, et al. Identifying EGFR mutations in lung adenocarcinoma by noninvasive imaging using radiomics features and random forest modeling. Eur Radiol. 2019; 29: 4742-50. 


\section{Tables}

Table 1. Demographic and clinical characteristics of the patients 


\begin{tabular}{|c|c|c|c|}
\hline \multirow[t]{2}{*}{ Characteristic } & Training cohort & Validation cohort & $p$-value \\
\hline & $(n=64)$ & $(\mathrm{n}=28)$ & \\
\hline Sex,n. (\%) & & & 0.086 \\
\hline Female & $14 \rrbracket 21.88 \rrbracket$ & $11 \rrbracket 39.29)$ & \\
\hline Male & $50 \otimes 78.12 \rrbracket$ & $17(60.71)$ & \\
\hline Age(years), mean (SD) & $20.44(8.98)$ & $20.04(8.99)$ & 0.844 \\
\hline Smoking status, n. (\%) & & & 0.656 \\
\hline No & $34(53.10)$ & $17(60.7)$ & \\
\hline Yes & $30(46.90)$ & $11(39.3)$ & \\
\hline Family history, n. (\%) & & & 0.754 \\
\hline No & $62(96.90)$ & $26(92.9)$ & \\
\hline Yes & $2(3.10)$ & $2(7.1)$ & \\
\hline TTF-1, n. (\%) & & & 0.070 \\
\hline Negative & $42(65.60)$ & $12(42.9)$ & \\
\hline Positive & $22(34.40)$ & $16(57.1)$ & \\
\hline Ki-67, n. (\%) & & & 0.560 \\
\hline Low expression & $33(51.60)$ & $17(60.7)$ & \\
\hline High expression & $31(48.40)$ & $11(39.3)$ & \\
\hline Histologic type, n. (\%) & & & 0.120 \\
\hline Adenocarcinoma & $32(50.00)$ & $20(71.43)$ & \\
\hline Squamous cell carcinoma & $28(43.80)$ & $6(21.43)$ & \\
\hline NOS & $4(6.20)$ & $2(7.14)$ & \\
\hline Stage, n. (\%) & & & 0.257 \\
\hline Ш & $23(35.90)$ & $6(21.40)$ & \\
\hline IV & $41(64.10)$ & $22(78.60)$ & \\
\hline T stage, n. (\%) & & & 0.842 \\
\hline 0 & $1(1.60)$ & $0(0.00)$ & \\
\hline 1 & $8(12.50)$ & $3(10.70)$ & \\
\hline 2 & $20(31.20)$ & $11(39.30)$ & \\
\hline
\end{tabular}




\begin{tabular}{|c|c|c|c|}
\hline 3 & 9 (14.10) & $5(17.90)$ & \\
\hline 4 & $26(40.60)$ & $9(32.10)$ & \\
\hline N stage, n. (\%) & & & 0.821 \\
\hline 0 & $6(9.38)$ & $3(10.70)$ & \\
\hline 1 & $5(7.81)$ & $1(3.60)$ & \\
\hline 2 & $25(39.06)$ & $13(46.40)$ & \\
\hline 3 & $28(43.75)$ & $11(39.30)$ & \\
\hline M stage, n. (\%) & & & 0.176 \\
\hline 0 & $22(34.40)$ & $5(17.90)$ & \\
\hline 1 & $42(65.60)$ & $23(82.10)$ & \\
\hline Lymph node metastasis, n. (\%) & & & 0.880 \\
\hline No & $9(14.10)$ & $5(17.90)$ & \\
\hline Yes & $55(85.90)$ & $23(82.10)$ & \\
\hline Intrapulmonary metastasis , n. (\%) & & & 0.466 \\
\hline No & $39(60.90)$ & $20(71.40)$ & \\
\hline Yes & $25(39.10)$ & $8(28.60)$ & \\
\hline Brain metastasis , n. (\%) & & & 0.330 \\
\hline No & $55(85.90)$ & $21(75.00)$ & \\
\hline Yes & $9(14.10)$ & $7(25.00)$ & \\
\hline Liver metastasis , n. (\%) & & & 1.000 \\
\hline No & $59(92.20)$ & $026(92.90)$ & \\
\hline Yes & $5(7.80)$ & $2(7.10)$ & \\
\hline Bone metastasis n. (\%) & & & 1.000 \\
\hline No & $44(68.80)$ & $019(67.90)$ & \\
\hline Yes & $20(31.20)$ & $9(32.10)$ & \\
\hline Pleural metastasis n. (\%) & & & 0.073 \\
\hline No & $54(84.38)$ & $19(67.86)$ & \\
\hline Yes & $10(15.62)$ & $9(32.14)$ & \\
\hline White blood cell, (median [IQR]) & $6.40[4.47,7.78]$ & $6.15[5.00,8.33]$ & 0.333 \\
\hline
\end{tabular}




\begin{tabular}{|lccc|} 
Neutrophil, (median [IQR]) & $69.05[62.65,73.78]$ & $65.20[58.50,71.08]$ & 0.203 \\
\hline Monocyte, (median [IQR]) & $8.65[6.83,11.03]$ & $6.70[5.27,9.45]$ & 0.952 \\
\hline CRP, (median [IQR]) & $18.10[5.05,19.85]$ & $12.00[1.17,18.83]$ & 0.330 \\
\hline CEA, (median [IQR]) & $13.60[3.88,84.30]$ & $11.30[2.80,84.30]$ & 0.568 \\
\hline NSE, (median [IQR]) & $14.25[10.35,19.10]$ & $15.05[12.00,19.10]$ & 0.513 \\
\hline
\end{tabular}

Abbreviations: CRP, C-reactive protein; CEA, carcinoembryonic antigen; NSE, neuron-specific enolase; NOS, not otherwise specified.

Table 2. Multivariable logistic regression analysis for nomogram model construction

\begin{tabular}{|lllll|}
\hline & Odds ratio & $95 \% \mathrm{Cl}$ & $\boldsymbol{P}$ \\
\hline & & Lower & Upper & \\
\hline Rad score & $1.270110^{5}$ & 3.832 & $3.418110^{8}$ & $<0.001$ \\
\hline Age & 0.910 & 0.184 & 0.989 & 0.045 \\
\hline Stage $\mathrm{n} 1$ & 0.075 & $5.880110^{-5}$ & 4.0456 & 0.260 \\
\hline Stage $\mathrm{n} 2$ & 6.030 & 0.421 & 86.171 & 0.171 \\
\hline Stage $\mathrm{n} 3$ & 1.990 & 0.139 & 23.380 & 0.584 \\
\hline Stage M & 0.153 & 0.022 & 0.777 & 0.035 \\
\hline
\end{tabular}

Abbreviation: OR, odds ratio.

Table 3. Predictive performance of the two models in the training and validation cohorts

\begin{tabular}{|c|c|c|c|c|c|c|}
\hline \multirow[t]{3}{*}{ Models } & \multicolumn{3}{|c|}{ Trainning cohort } & \multicolumn{3}{|l|}{ Validation cohort } \\
\hline & \multicolumn{3}{|c|}{$\begin{array}{l}\text { AUC Sensitivity Specificity } \\
\text { Accuracy }\end{array}$} & \multirow{2}{*}{$\begin{array}{l}\text { AUC } \\
(95 \% \mathrm{Cl})\end{array}$} & \multicolumn{2}{|c|}{$\begin{array}{l}\text { Sensitivity } \\
\text { Specificity Accuracy }\end{array}$} \\
\hline & $(95 \% \mathrm{Cl})$ & $(\%) \quad(\%)$ & (\%) & & (\%) & $(\%) \quad(\%)$ \\
\hline $\begin{array}{l}\text { Radiomic } \\
0.848(0.7\end{array}$ & $\begin{array}{l}\text { del1 } \\
\text { 952) }\end{array}$ & $\begin{array}{l}0.6250 .906 \\
1.000)\end{array}$ & 0.766 & $0.795(0.581-$ & 1.000 & $\begin{array}{l}0.704 \\
0.714\end{array}$ \\
\hline \multicolumn{2}{|c|}{$\begin{array}{l}\text { Radiomics } \\
0.902(0.811-0.994) \\
\text { nomogram model1 }\end{array}$} & $\begin{array}{l}0.8570 .884 \\
1.000)\end{array}$ & 0.875 & 0.877(0.735- & 0.800 & $\begin{array}{l}0.944 \\
0.893\end{array}$ \\
\hline
\end{tabular}




\begin{tabular}{|c|c|c|c|c|}
\hline & $\mathrm{HR}$ & $95 \% \mathrm{Cl}$ & & $P$ \\
\hline & & Lower & Upper & \\
\hline Rad score & 0.005 & $4.572 * 10^{-3}$ & 0.043 & $<0.001$ \\
\hline CRP & 1.015 & 0.996 & 1.035 & 0.130 \\
\hline M stage & 2.449 & 1.138 & 5.269 & 0.020 \\
\hline
\end{tabular}

Abbreviations: HR, hazard ratio; CRP, C-reactive protein

Table 5. Harrell's concordance indexes for the different modalities

\begin{tabular}{|lclll|}
\hline Modalities & Training cohort(n=64) & $\begin{array}{l}\text { Validation } \\
\text { cohort }(\mathrm{n}=28)\end{array}$ & \\
& C-index $95 \% \mathrm{Cl}$ & $\begin{array}{l}\text { Brier } \\
\text { Score }\end{array}$ & C-index 95\% Cl & Brier score \\
\hline Radiomics model2 & $0.717(0.612,0.822)$ & 0.178 & $0.760(0.574,0.946)$ & 0.187 \\
\hline $\begin{array}{l}\text { Radiomics nomogram } \\
\text { mode2 }\end{array}$ & $0.749(0.643,0.854)$ & 0.187 & $0.791(0.605,0.978)$ & 0.209 \\
\hline $\begin{array}{l}\text { Abbreviations: C-index, Harrell's concordance } \\
\text { index }\end{array}$ & & & & \\
\hline
\end{tabular}

\section{Figures}




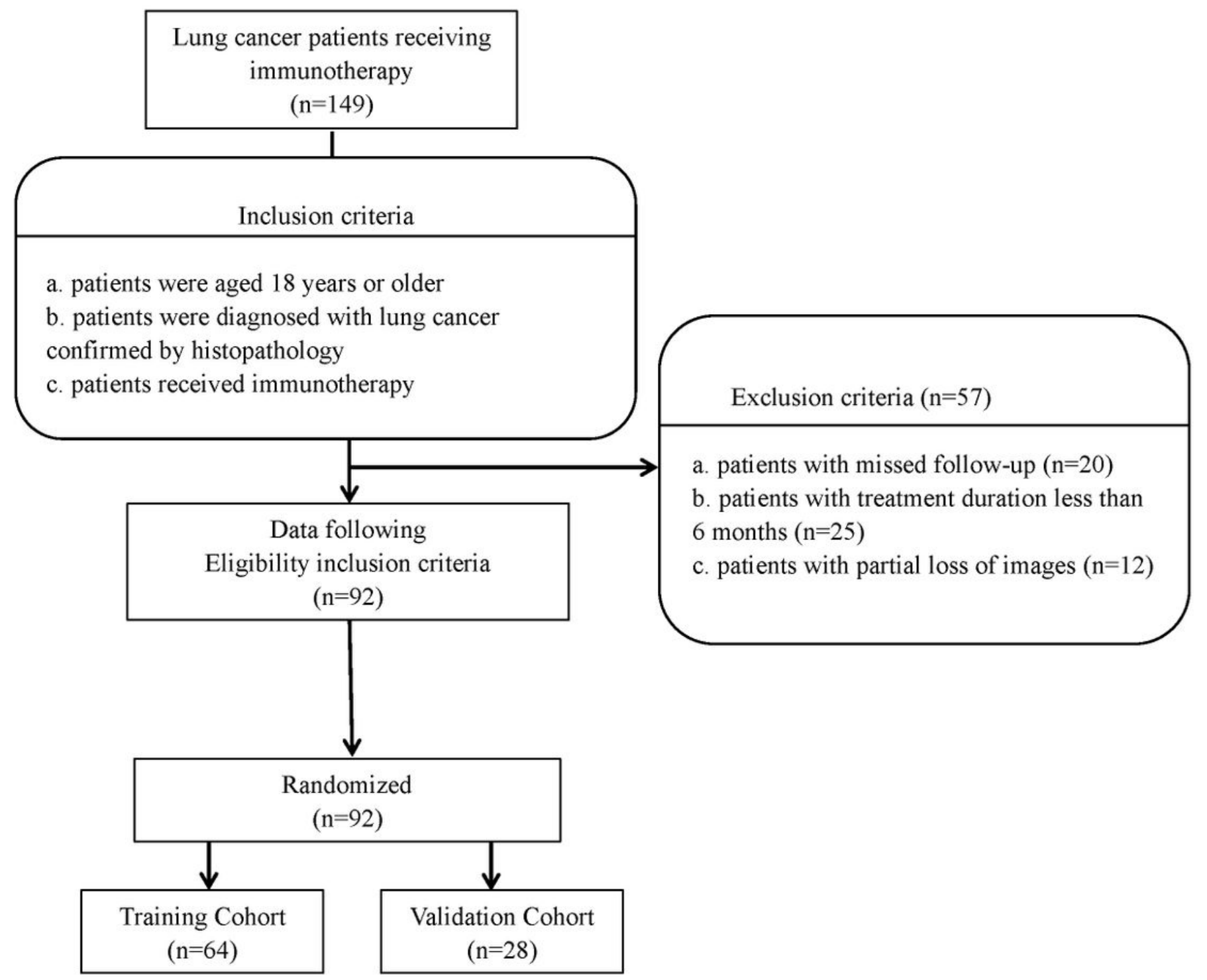

Figure 1

Flow diagram of the enrollment of patients with non-small cell lung cancer. 


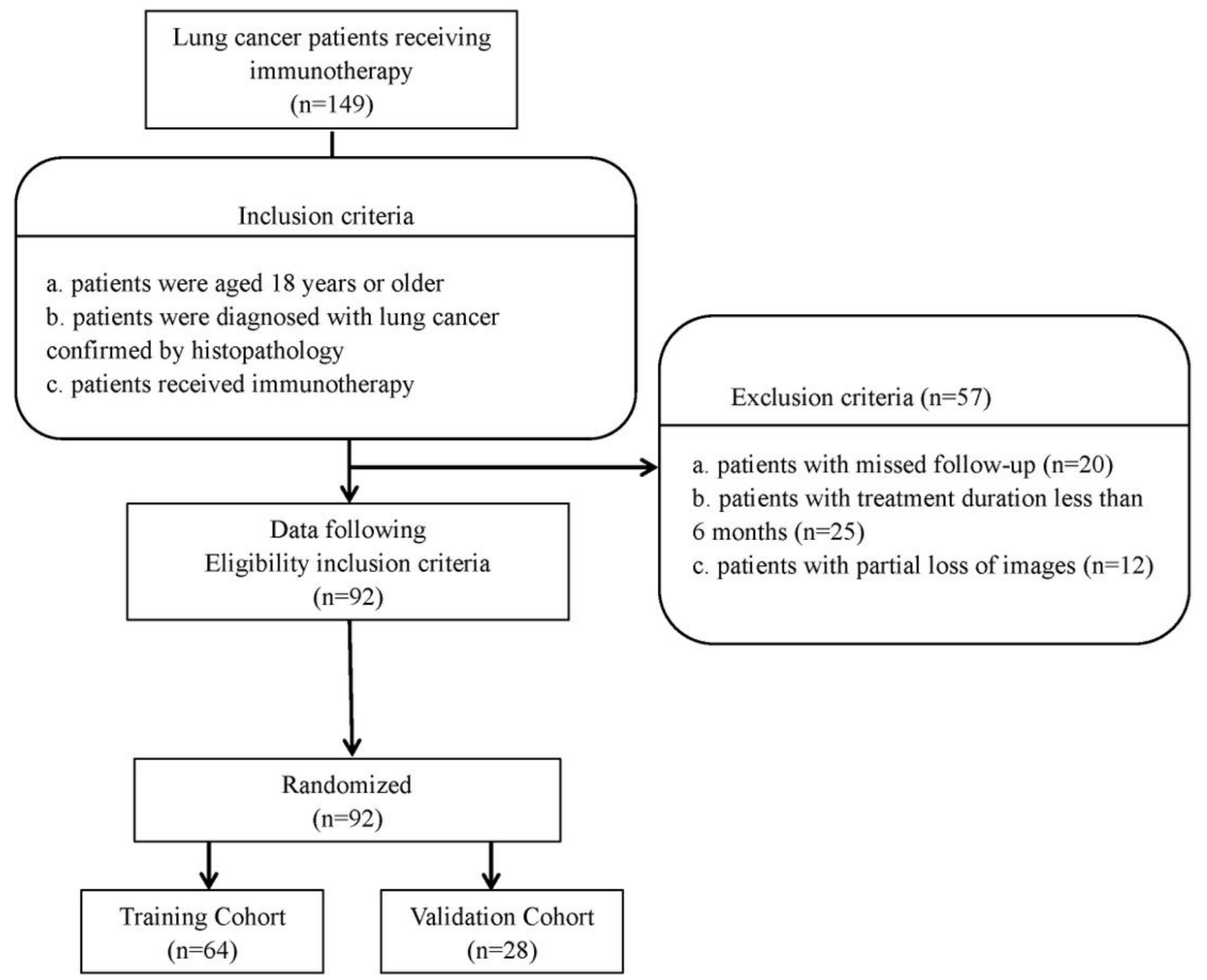

Figure 1

Flow diagram of the enrollment of patients with non-small cell lung cancer. 


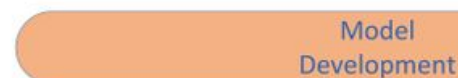

Development

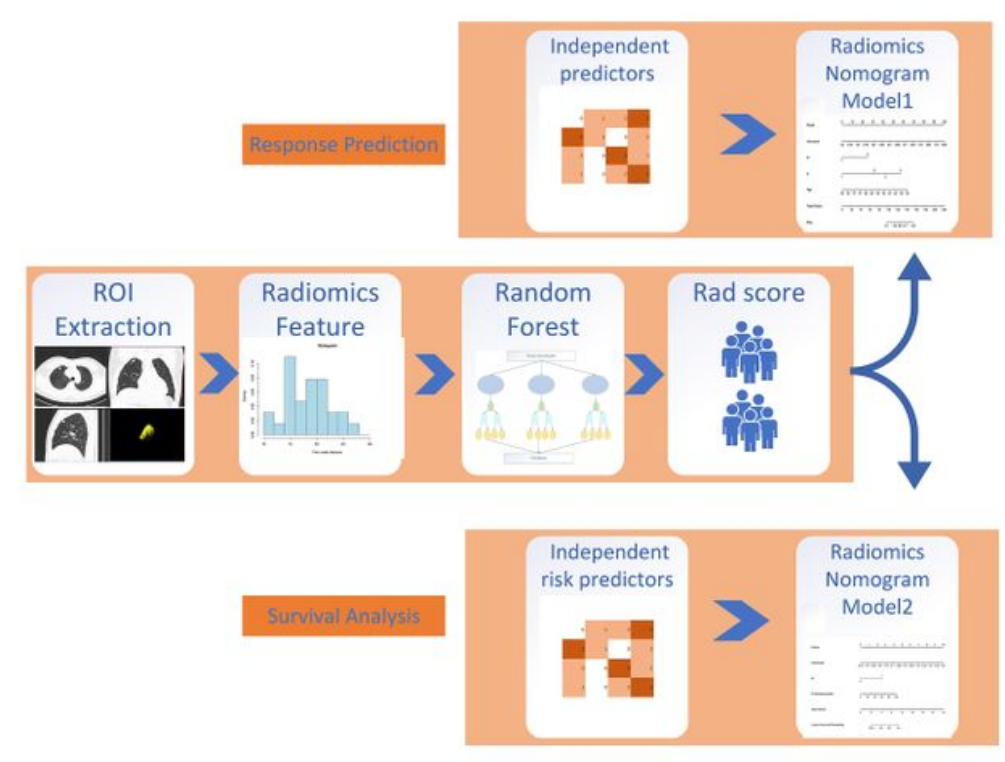

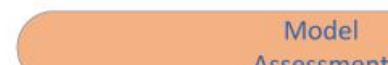

Assessment
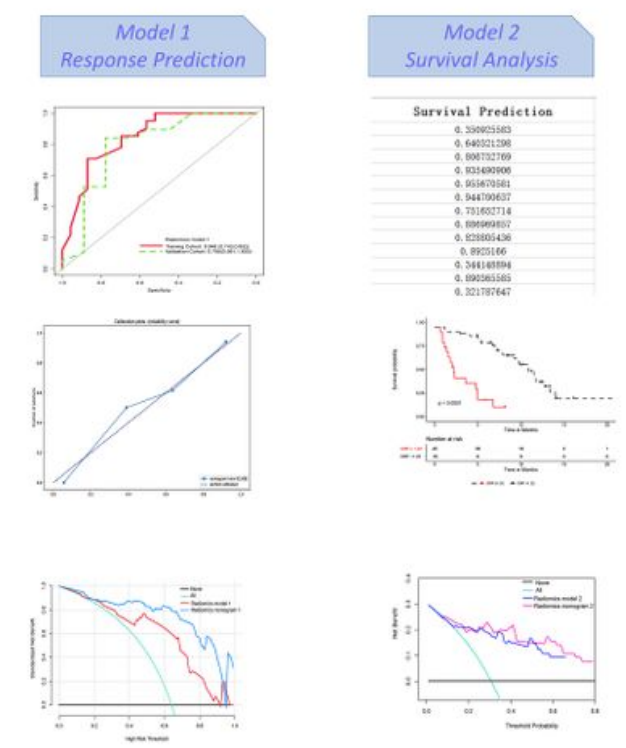

\section{Figure 2}

Workflow for developing the radiomics nomogram models. CT image segmentation was performed using manual semiautomatic segmentation in radiomics prototype software (Radiomics, Frontier, Siemens). The radiomic features from the volumes of interest were then computed with CT images on the prototype. A predictive model was constructed on the basis of the CT-derived radiomic features using the random forest (RF) method to output a radiomics score (rad-score) for each patient. The rad-score was combined with significant clinicopathological factors for multivariate logistic regression analysis to develop radiomics nomogram model 1 for predicting the durable clinical benefit (DCB). Radiomics nomogram model 2 for predicting progression-free survival (PFS) was developed through the multivariate logistic regression analysis of the rad-score and independent risk factors combined. 


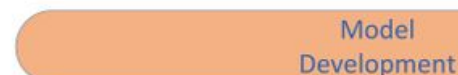

Development
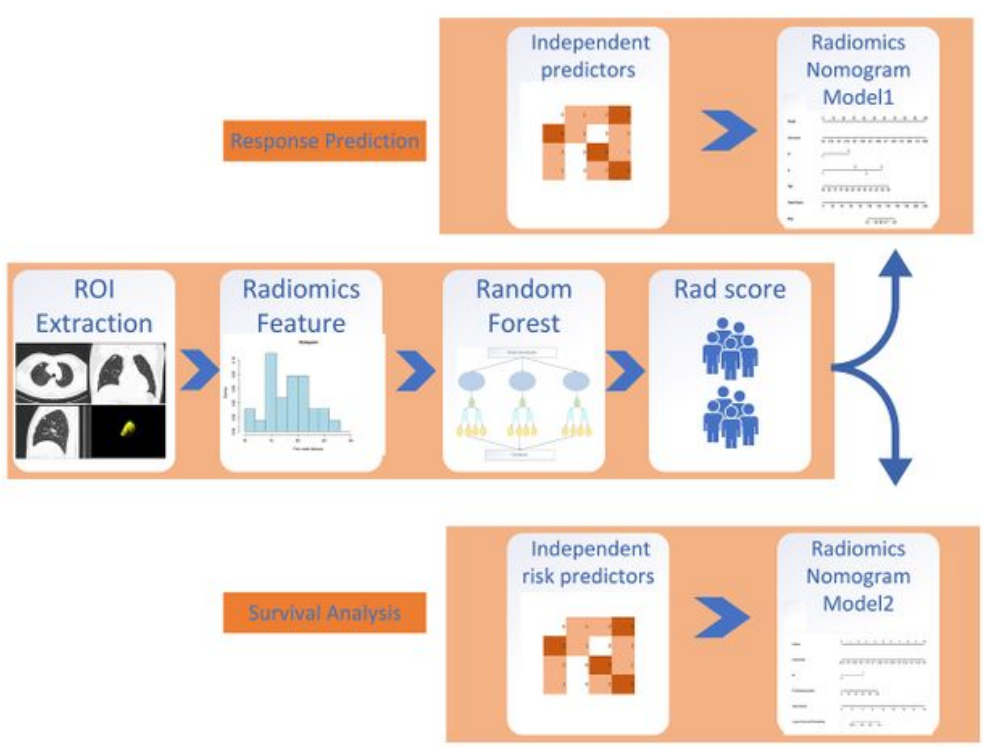

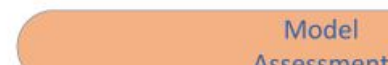

Assessment
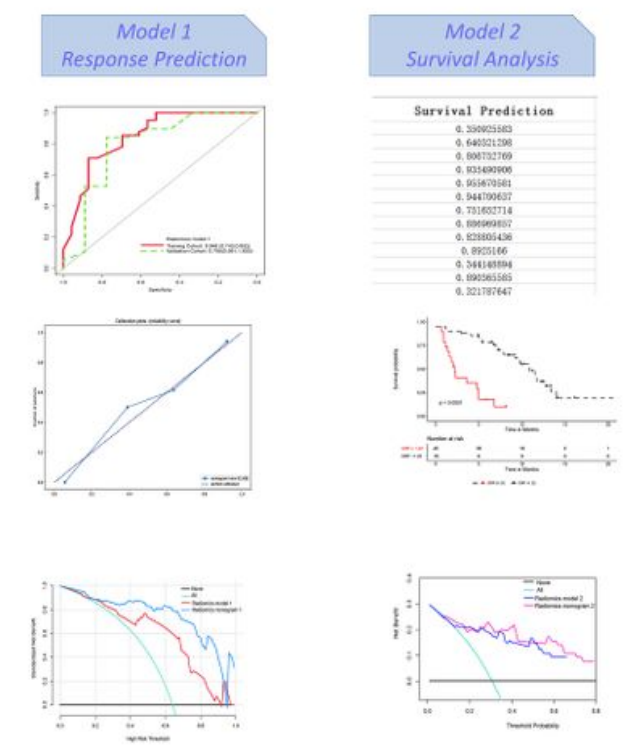

\section{Figure 2}

Workflow for developing the radiomics nomogram models. CT image segmentation was performed using manual semiautomatic segmentation in radiomics prototype software (Radiomics, Frontier, Siemens). The radiomic features from the volumes of interest were then computed with CT images on the prototype. A predictive model was constructed on the basis of the CT-derived radiomic features using the random forest (RF) method to output a radiomics score (rad-score) for each patient. The rad-score was combined with significant clinicopathological factors for multivariate logistic regression analysis to develop radiomics nomogram model 1 for predicting the durable clinical benefit (DCB). Radiomics nomogram model 2 for predicting progression-free survival (PFS) was developed through the multivariate logistic regression analysis of the rad-score and independent risk factors combined. 

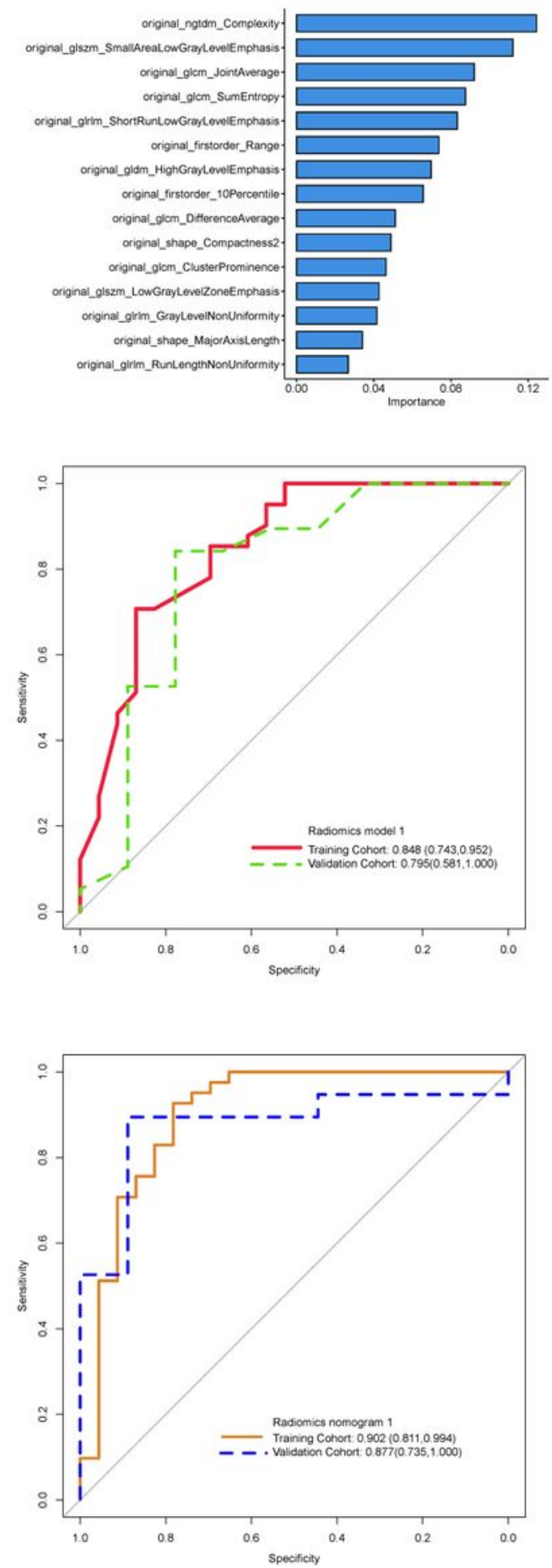

\section{Figure 3}

Receiver operating characteristic curves for the different models. (A) 15 radiomic were screened for the prediction model. Receiver operating characteristic curves for the different models. (B) Receiver operating characteristic curves showing the differences between the training cohort and validation cohort in radiomics model 1 . Receiver operating characteristic curves for the different models. (C) Receiver 
operating characteristic curves showing the differences between the training cohort and validation cohort in radiomics nomogram model 1.
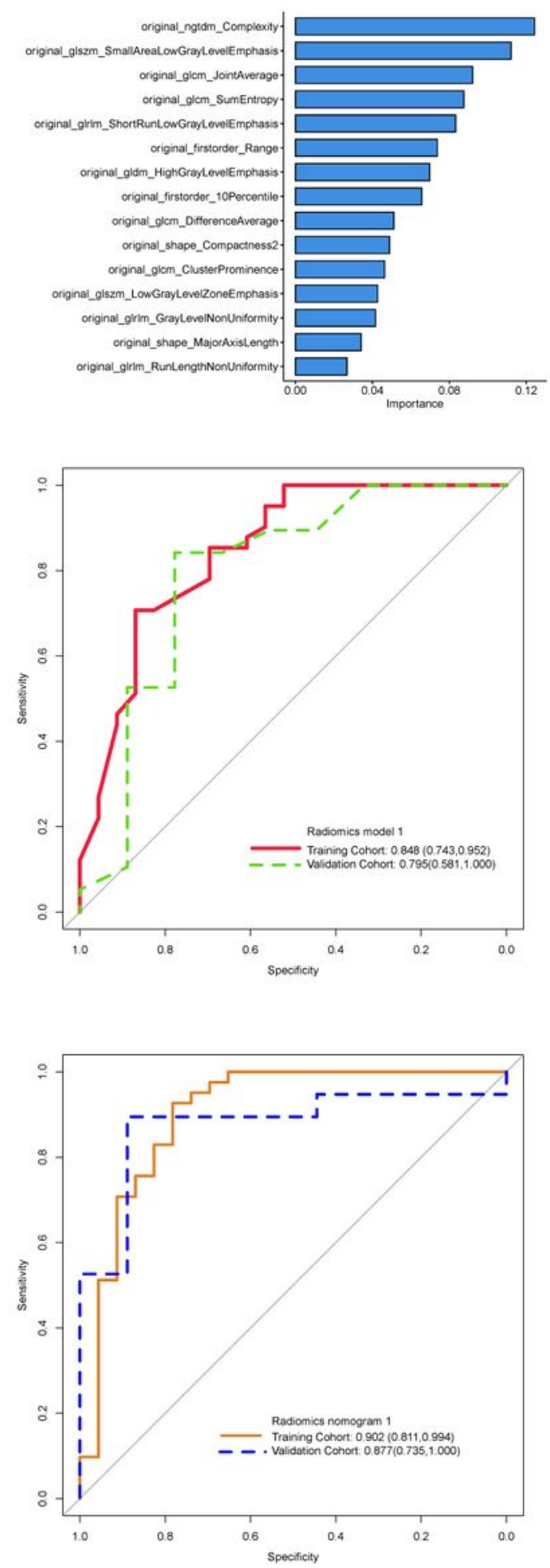

Figure 3

Receiver operating characteristic curves for the different models. (A) 15 radiomic were screened for the prediction model. Receiver operating characteristic curves for the different models. (B) Receiver operating characteristic curves showing the differences between the training cohort and validation cohort in 
radiomics model 1 . Receiver operating characteristic curves for the different models. (C) Receiver operating characteristic curves showing the differences between the training cohort and validation cohort in radiomics nomogram model 1.
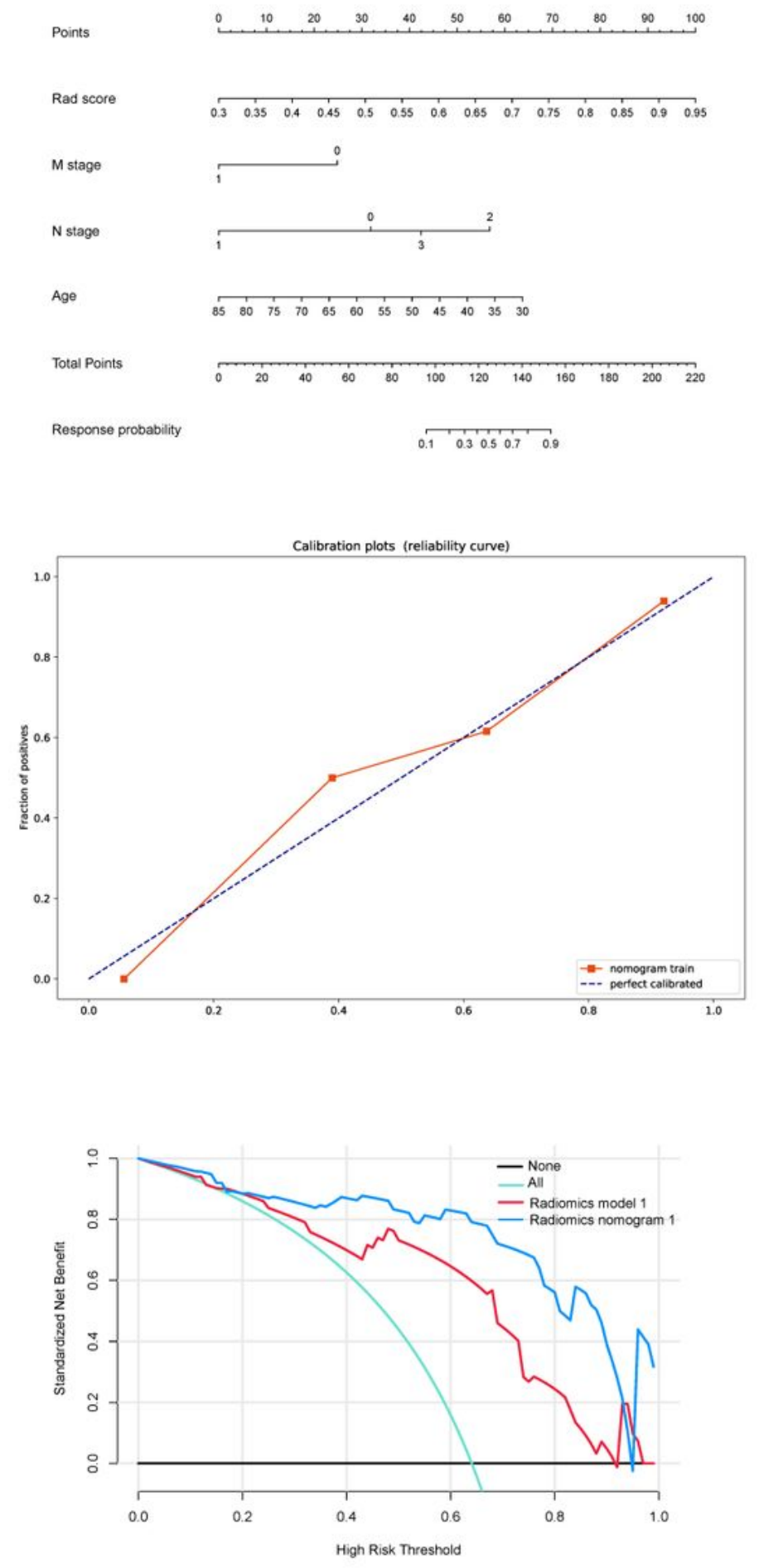

\section{Figure 4}

Development of radiomics nomogram model 1. (A) Nomogram based on independent predictors (radscore, age, stage $n 1$, stage $n 2$, stage $n 3$, and stage M). Development of radiomics nomogram model 1. (B) 
Calibration curves of the nomogram in the training cohort. The horizontal axis is the predicted incidence of the durable clinical benefit (DCB), whereas the vertical axis is the observed incidence of the DCB. The dotted line on the diagonal is the reference line at which the predicted value is equal to the actual value. The orange line is the calibration curve. Development of radiomics nomogram model 1. (C) Decision curve analysis for each model. The y-axis measures the net benefit, which was calculated using truepositive and false-positive results. Radiomics nomogram model 1 had the highest net benefit among all positive predictions (line labeled "All"), all negative predictions (line labeled "None"), and models (line labeled "radiomics model 1 ") at the threshold from 0.1 to 0.9 . 

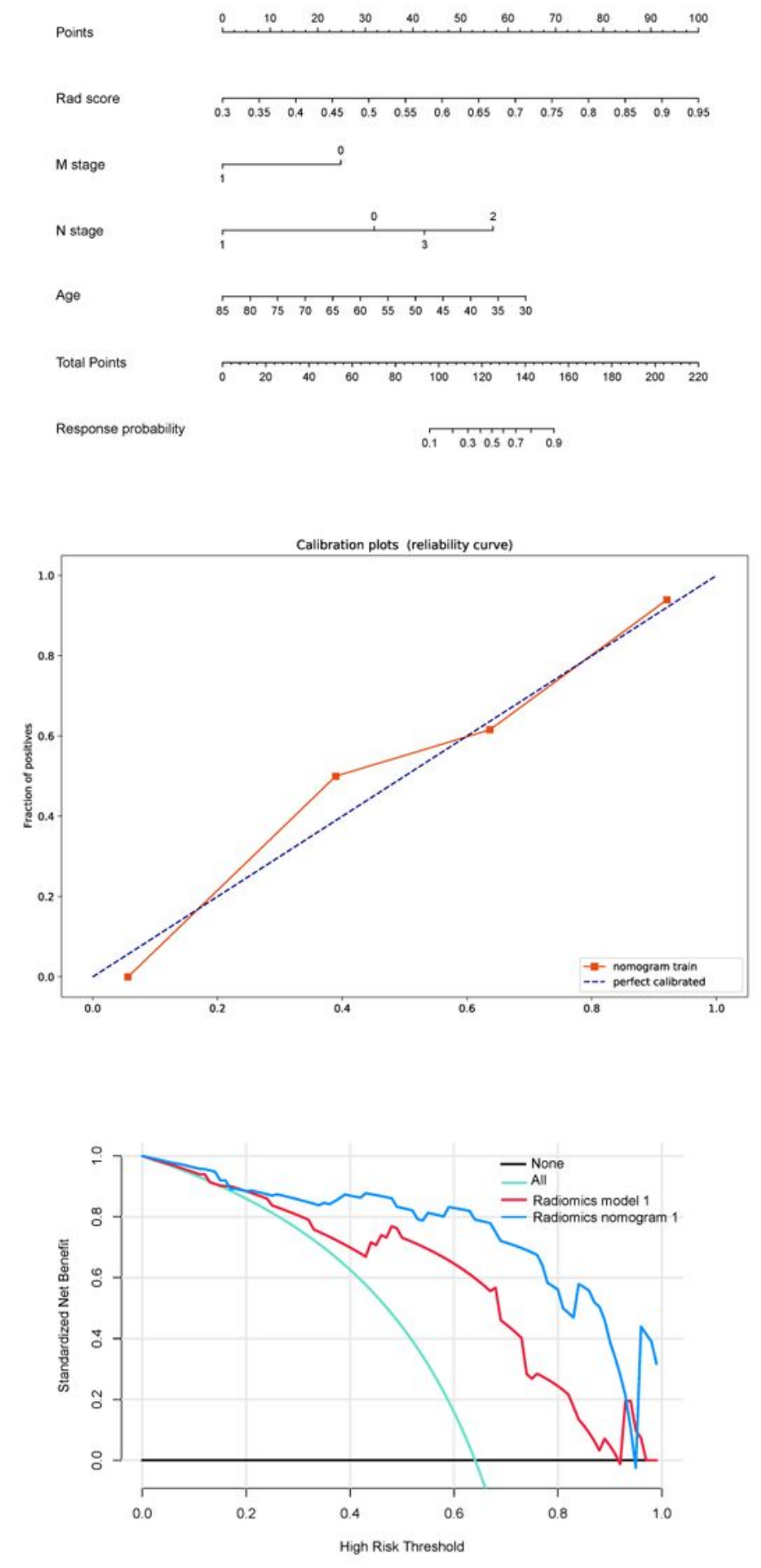

\section{Figure 4}

Development of radiomics nomogram model 1. (A) Nomogram based on independent predictors (radscore, age, stage $n 1$, stage $n 2$, stage $n 3$, and stage M). Development of radiomics nomogram model 1. (B) Calibration curves of the nomogram in the training cohort. The horizontal axis is the predicted incidence of the durable clinical benefit (DCB), whereas the vertical axis is the observed incidence of the DCB. The dotted line on the diagonal is the reference line at which the predicted value is equal to the actual value. 
The orange line is the calibration curve. Development of radiomics nomogram model 1. (C) Decision curve analysis for each model. The y-axis measures the net benefit, which was calculated using truepositive and false-positive results. Radiomics nomogram model 1 had the highest net benefit among all positive predictions (line labeled "All"), all negative predictions (line labeled "None"), and models (line labeled "radiomics model 1") at the threshold from 0.1 to 0.9 .
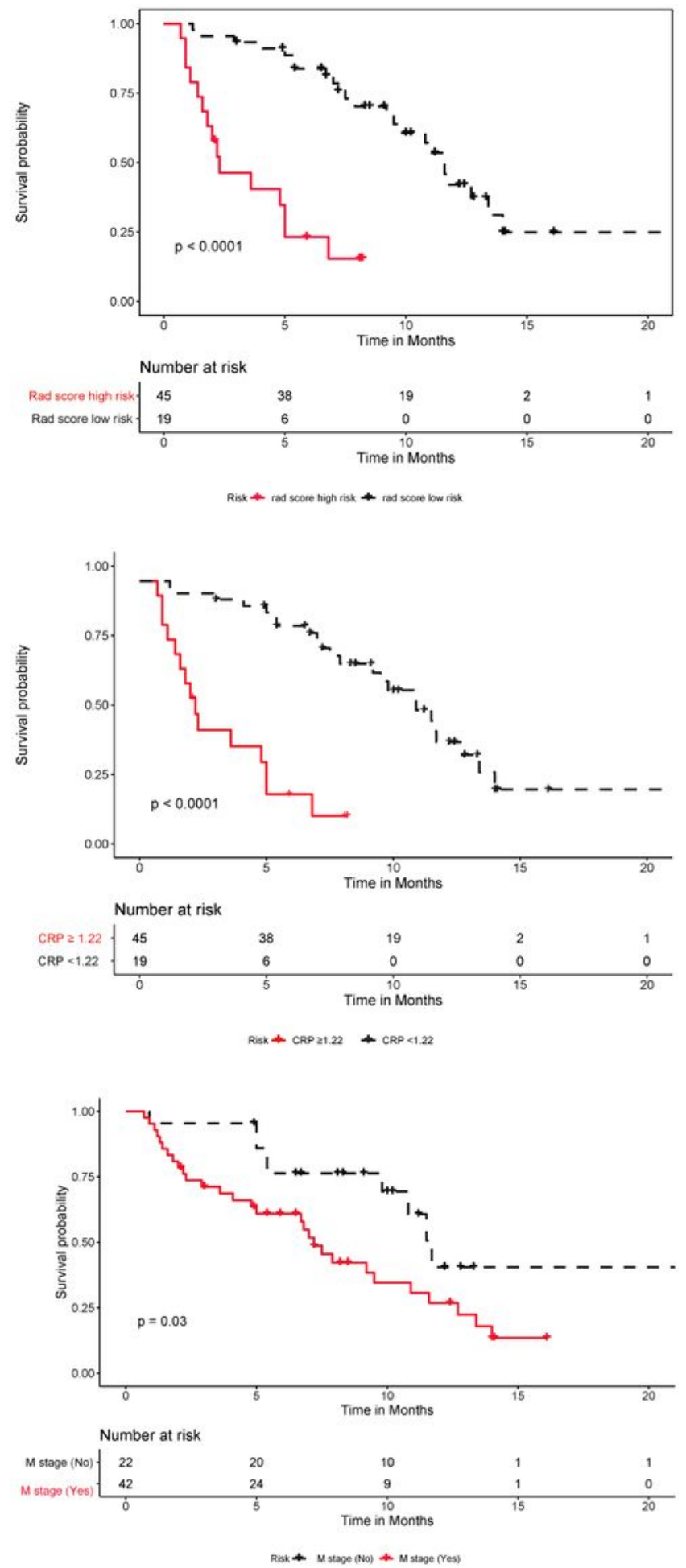

Figure 5 
Predictive performances of the rad-score, C-reactive protein (CRP) level, and stage $M$ in the Kaplan-Meier survival analysis of patients in the high- and low-risk groups in the training cohort. Kaplan-Meier analysis for the rad-score $(A)$. The patients were stratified into high- and low-risk groups on the basis of the radscore (A, $p<0.0001$, log-rank test) Predictive performances of the rad-score, C-reactive protein (CRP) level, and stage $M$ in the Kaplan-Meier survival analysis of patients in the high- and low-risk groups in the training cohort. Kaplan-Meier analysis for the CRP level (B). The patients were stratified into high- and low-risk groups on the basis of CRP level (B, $p<0.0001$, log-rank test). Predictive performances of the radscore, $C$-reactive protein (CRP) level, and stage $M$ in the Kaplan-Meier survival analysis of patients in the high- and low-risk groups in the training cohort. Kaplan-Meier analysis for stage $M(C)$. The patients were stratified into high- and low-risk groups on the basis of stage $M(C, p=0.03$, log-rank test). 

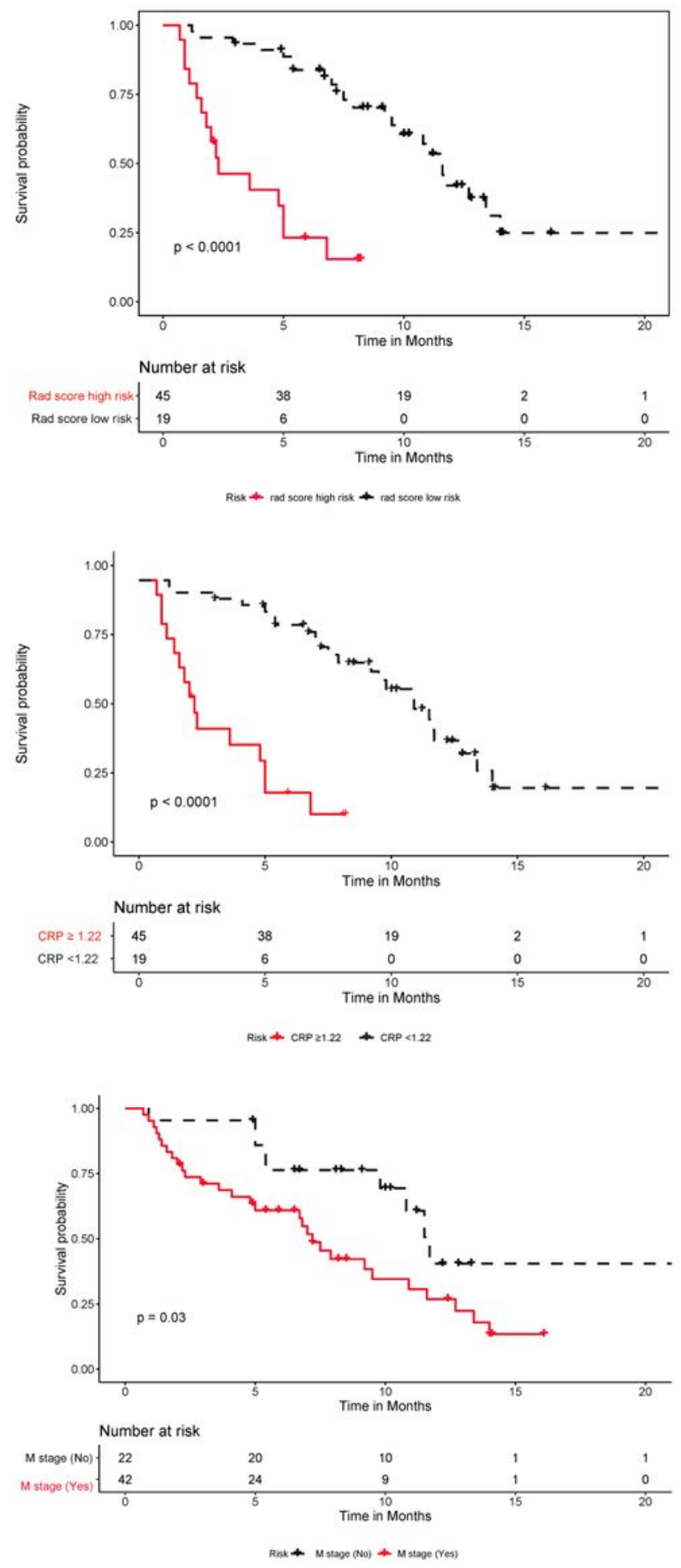

\section{Figure 5}

Predictive performances of the rad-score, C-reactive protein (CRP) level, and stage $\mathrm{M}$ in the Kaplan-Meier survival analysis of patients in the high- and low-risk groups in the training cohort. Kaplan-Meier analysis for the rad-score $(A)$. The patients were stratified into high- and low-risk groups on the basis of the radscore (A, $p<0.0001$, log-rank test) Predictive performances of the rad-score, C-reactive protein (CRP) level, and stage $\mathrm{M}$ in the Kaplan-Meier survival analysis of patients in the high- and low-risk groups in the 
training cohort. Kaplan-Meier analysis for the CRP level (B). The patients were stratified into high- and low-risk groups on the basis of CRP level (B, $p<0.0001$, log-rank test). Predictive performances of the radscore, C-reactive protein (CRP) level, and stage $\mathrm{M}$ in the Kaplan-Meier survival analysis of patients in the high- and low-risk groups in the training cohort. Kaplan-Meier analysis for stage $\mathrm{M}$ (C). The patients were stratified into high- and low-risk groups on the basis of stage $M(C, p=0.03$, log-rank test).

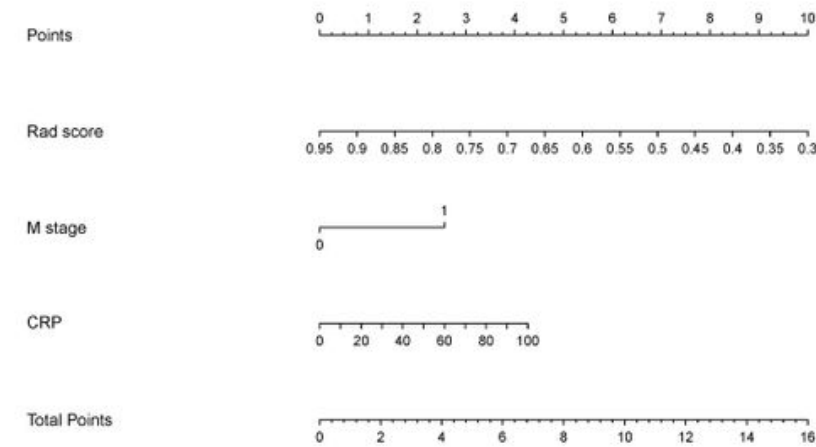

2 year Survival Probability
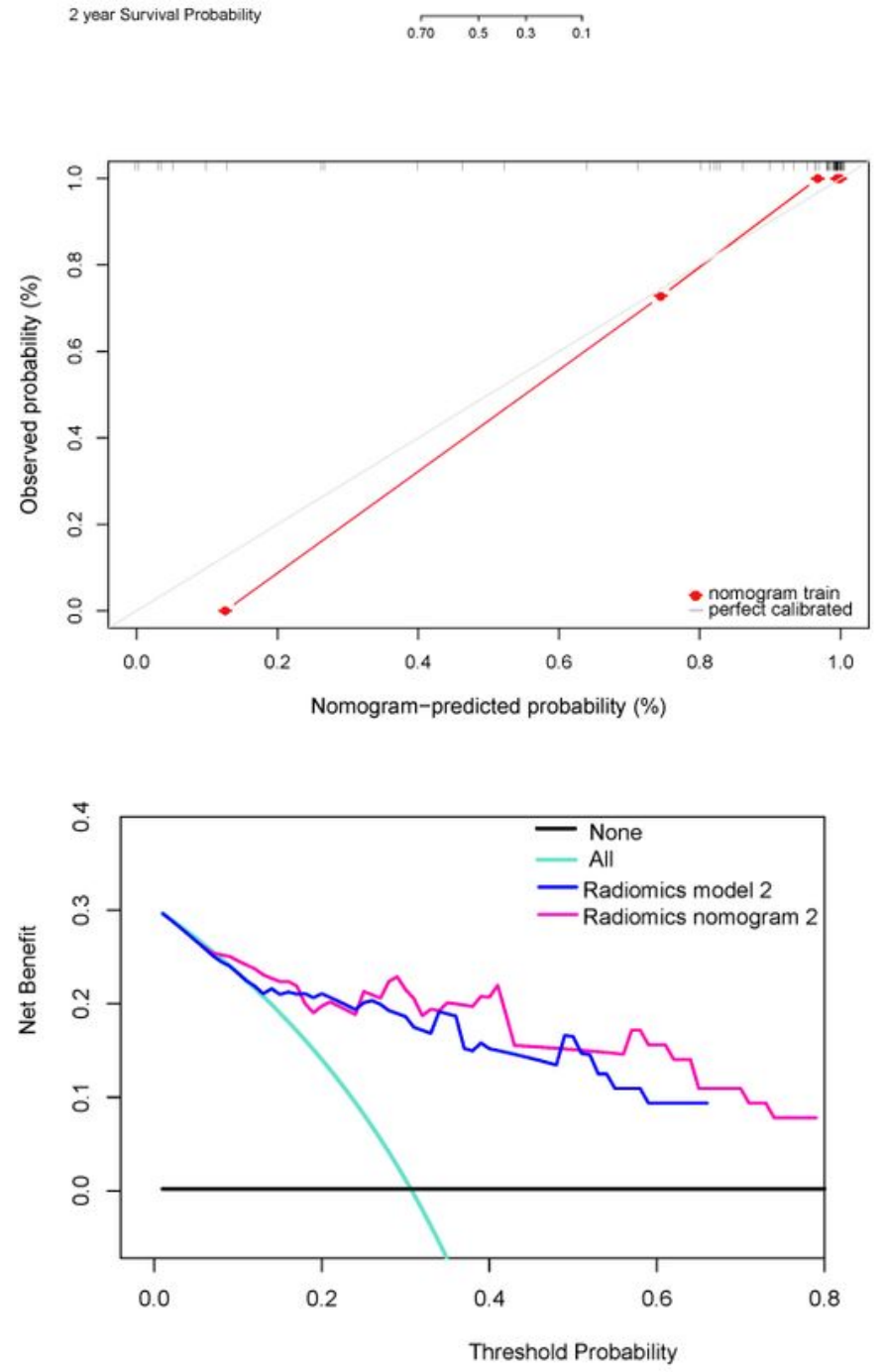

Figure 6 
Development of radiomics nomogram model 2. (A) Nomogram based on independent predictors (radscore, stage $\mathrm{M}$ disease, and C-reactive protein (CRP) level). Development of radiomics nomogram model 2. (B) Calibration curves of the nomogram in the training cohort. The horizontal axis is the predicted incidence of progression-free survival (PFS), whereas the vertical axis is the observed incidence of PFS. The gray line on the diagonal is the reference line at which the predicted value is equal to the actual value. The red line is the calibration curve. Development of radiomics nomogram model 2. (C) Decision curve analysis for each model. The y-axis measures the net benefit, which was calculated using truepositive and false-positive results. Radiomics nomogram model 2 had the highest net benefit among all positive predictions (line labeled "All"), all negative predictions (line labeled "None"), and models (line labeled "radiomics model 2") at the threshold from 0.1 to 0.9 . 


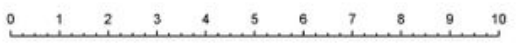

Rad score

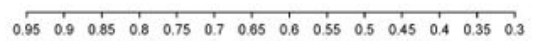

M stage

CRP

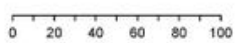

Total Points

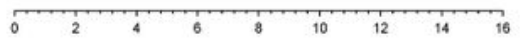

2 year Survival Probability
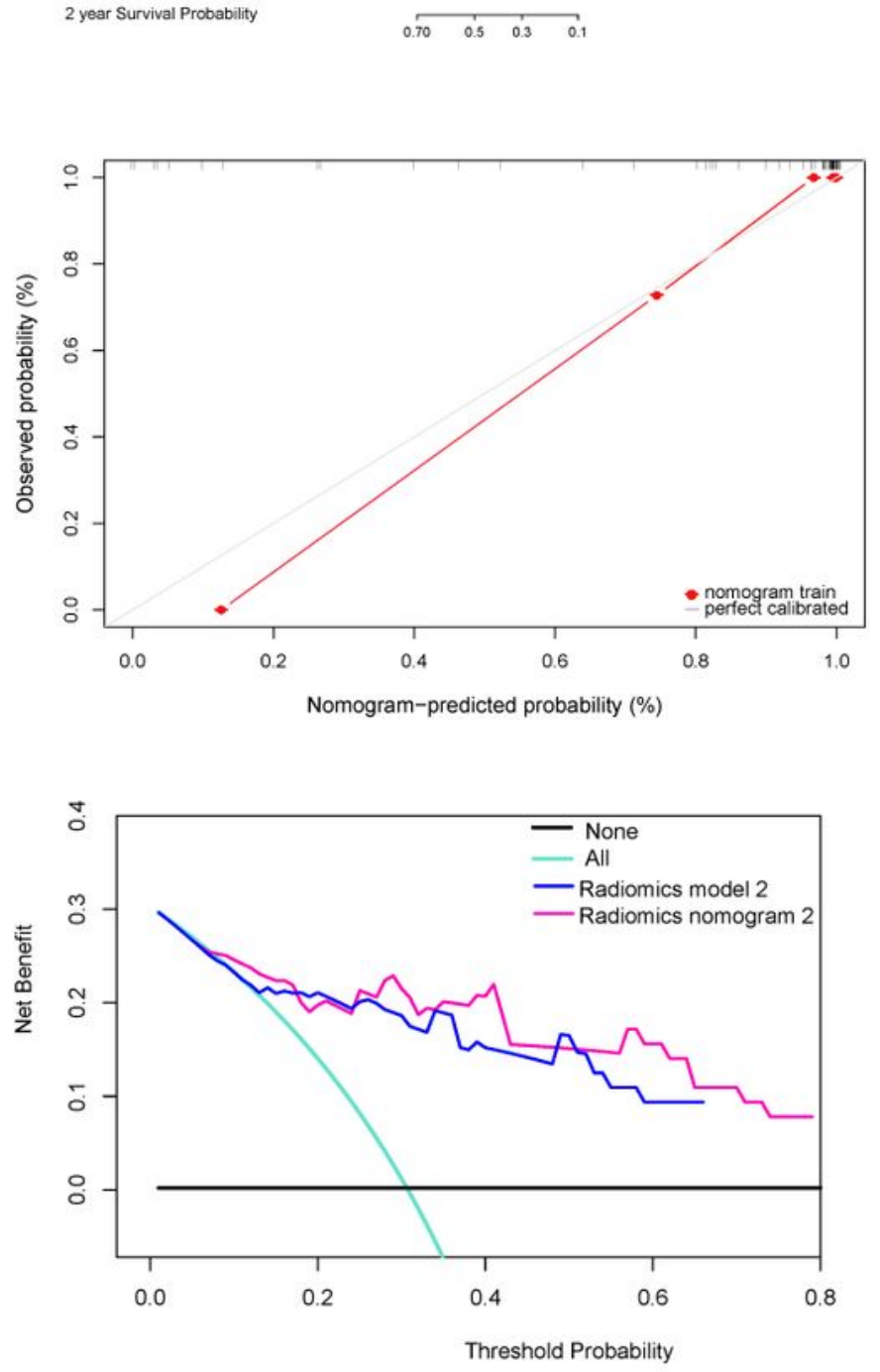

Figure 6

Development of radiomics nomogram model 2. (A) Nomogram based on independent predictors (radscore, stage $\mathrm{M}$ disease, and C-reactive protein (CRP) level). Development of radiomics nomogram model 2. (B) Calibration curves of the nomogram in the training cohort. The horizontal axis is the predicted incidence of progression-free survival (PFS), whereas the vertical axis is the observed incidence of PFS. The gray line on the diagonal is the reference line at which the predicted value is equal to the actual 
value. The red line is the calibration curve. Development of radiomics nomogram model 2. (C) Decision curve analysis for each model. The y-axis measures the net benefit, which was calculated using truepositive and false-positive results. Radiomics nomogram model 2 had the highest net benefit among all positive predictions (line labeled "All"), all negative predictions (line labeled "None"), and models (line labeled "radiomics model 2") at the threshold from 0.1 to 0.9 . 